\begin{tabular}{|c|l|}
\hline Title & Global Properties of Infectious Disease Models with Nonlinear Incidence \\
\hline Author(s) & Korobeinikov, A ndrei \\
\hline Citation & $\begin{array}{l}\text { Bulletin of Mathematical Biology, 69/6), 1871-1886 } \\
\text { https://doi.org/10.1007/311538-007-9196-y }\end{array}$ \\
\hline Issue Date & 2007-08 \\
\hline Doc URL & http://hdl.handle.net/2115/30189 \\
\hline Rights & $\begin{array}{l}\text { The original publication is available at } \\
\text { www.springerlink.com }\end{array}$ \\
\hline Type & article (author version) \\
\hline File Information & BMB69-6.pdf \\
\hline
\end{tabular}

Instructions for use 


\title{
GLOBAL PROPERTIES OF INFECTIOUS DISEASE MODELS WITH NON-LINEAR INCIDENCE
}

\author{
ANDREI KOROBEINIKOV
}

Laboratory of Nonlinear Science and Computation

Research Institute for Electronic Science, Hokkaido University,

Sapporo 060-0812, Japan

andrei@nsc.es.hokudai.ac.jp

phone (81 11) 7062414

fax (81 11) 7064966

AMS Classification

92D30 (primary), 34D20 (secondary)

\section{Proposed Running Head}

Models with non-linear incidence 
Abstract. We consider global properties for the classical $S I R$, $S I R S$ and $S E I R$ models of infectious diseases, including the models with the vertical transmission, assuming that the horizontal transmission is governed by an unspecified function $f(S, I)$. We construct Lyapunov functions which enable us to find biologically realistic conditions sufficient to ensure existence and uniqueness of a globally asymptotically stable equilibrium state. This state can be either endemic, or infection-free, depending on the value of the basic reproduction number.

Key words: Direct Lyapunov method, Lyapunov function, endemic equilibrium state, global stability, nonlinear incidence.

Acknowledgement. This research is supported by Japan Society for the Promotion of Science, through Project 17540099.

\section{INTRODUCTION}

It is traditionally postulated that the spread of an infectious disease in a population occurs according to the principle of mass action. This assumption implies that the corresponding incidence rate is bilinear with respect to the numbers of susceptible and infective individuals (Anderson and May, 1991; Hethcote, 2000); more specifically, if $S(t)$ and $I(t)$ are the fractions of susceptibles and infective individuals in a population, and if $\beta$ is the per capita contact rate, then the principle of mass action implies that the infection spreads with the rate $\beta I S$. The set of assumptions behind this postulate is fairly sound, and the models based on this postulate allowed to gain a number of important results. 
However, there is a number of reasons why this standard bilinear incidence rate may require modification. For instance, the underlying assumption of homogeneous mixing and homogeneous environment may be invalid. In this case the necessary population structure and heterogeneous mixing may be incorporated into a model with a specific form of non-linear transmission. Saturation effects also lead to a nonlinear incidence rate: if the portion of the infective hosts in a population is very high and exposure to the disease agent is virtually certain, then the transmission rate may grow more slowly than linear with the increase in the number of infectives. This effect was encountered in clinical observations as well as in laboratory experiments (Capasso and Serio, 1978; Brawn and Hasibuan, 1995). Furthermore, the details of transmission of infectious diseases are generally unknown, and may vary for different conditions; this observation justifies the growing interest to the models with incidence rates of more general form.

For this reason models of infectious diseases with nonlinear incidence rates have been attracting considerable attention over the last two decades. The most common in the literature nonlinear incidence rate takes the form $\beta I^{p} S^{q}$ (where $p$ and $q$ are positive constants). Models with this incidence rate have been studied by Liu, Levin and Iwasa (1986) and Liu, Hethcote and Levin (1987), and later by Derrick and van den Driessche (2003); Hethcote, Lewis and van den Driessche (1989); Hethcote and van den Driessche (1991); Korobeinikov and Maini (2004), and many others. The nonlinear incidence rates of more sophisticated forms were also suggested and studied. For instance, 
Liu, Levin and Iwasa (1986) proposed an incidence rate of the form $k I^{p} S /\left(1+\alpha I^{l}\right)$; Briggs and Godfray (1995), who studied infection of insects, considered a non-linear pathogen transmission of the form $k S \ln (1+\nu P / k)$ (where $P$ is the density of the pathogen particles). Li, Muldowney and van den Driessche (1999) studied the global properties of a $S E I R$ model with the incidence rate of the form $g(I) S$; they also extended some of their results for a $S E I R S$ model. Korobeinikov and Maini (2005) considered a variety of models with the incidence rate of the form $g(I) h(S)$; constructed Lyapunov functions enabled to establish global properties for a $S I R$ and a $S E I R$ models.

In a more general case, transmission of an infection may be given by a non-factorable function of $S$ and $I$. Feng and Thieme (2000) considered the most general model with arbitrarily many infective stages with general length distribution, and with incidence rate given by an arbitrary function $f\left(S, I_{1}, \ldots, I_{n}\right)$. They found the condition for existence and uniqueness of the endemic equilibrium state, and for its local stability. For a comparatively simple case of $S I R$ and $S I R S$ models with the incidence rate given by an unspecified function $f(S, I)$, sufficient conditions for the global stability of the endemic equilibrium state was also found (Korobeinikov, 2006).

The main goal of this notice is to study the global properties of the classical three-dimensional SEIR model (including the model with the vertical transmission) with the horizontal transmission governed by an unspecified function $f(S, I)$. For this rather general case, by means of the direct Lyapunov method, we derive sufficient conditions 
ensuring existence and uniqueness of a globally asymptotically stable steady state. It is not surprising, however, that the Lyapunov functions constructed here for the three-dimensional SEIR models can also be applied to the two-dimensional SIR and SIRS models. Although the properties of these models were already studied, for the sake of consistency we consider here these models as well. We have to stress, however, that the Lyapunov function constructed here is not a direct generalisation of the functions we apply earlier (Korobeinikov, 2006) to establish the global properties of the $S I R$ and $S I R S$ models.

\section{SIR AND SEIR MODELS}

We assume that the population size is constant, that is deaths are balanced by births. Following classical assumptions (Anderson and May, 1991; Busenberg and Cooke, 1993; Hethcote, 2000), we divide the host population into the susceptible, the infective and the recovered subpopulations, and denote the fractions of these in the population by $S, I$ and $R$ respectively (that is $S+I+R=1$ ). We assume that after infection an individual moves from the susceptibles compartment into the infectives compartment and then into the recovered compartment. If recovery implies permanent immunity, then a recovered individual remains in the recovered compartment; a model based on these assumptions is known as a $S I R$ model. If all the birth are into the susceptibles compartment, and if the transmission of the infection is governed by 
an incidence rate $f(S, I)$, then the basic $S I R$ model is

$$
\begin{aligned}
\dot{S} & =\mu-f(S, I)-\mu S, \\
\dot{I} & =f(S, I)-\delta I, \\
\dot{R} & =\sigma I-\mu R .
\end{aligned}
$$

Here $\delta=\sigma+\mu$ is the sum of the recovery rate and the death rate. If the transmission rate $f$ does not depend on the variable $R$, the third equation for the recovered population can be omitted.

Naturally, a biologically feasible incidence rate $f(S, I)$ is a positive monotonically growing function for all $S, I \geq 0$, and $f(0, I)=f(S, 0)=$ 0 . These conditions arise solely from biological considerations; further we will show that they are not necessary for our analysis. We also assume that this function is continuous and differentiable for all $S, I \geq$ 0.

For many infections, some time has to pass before an infected individual becomes infectious. The simplest way to consider this latent state is to add an extra class, the class of exposed hosts, to the system. Let us assume now that $S, E, I$ and $R$ are respectively the fractions of the susceptibles, the exposed, the infectives and the recovered hosts in the population, that is $S+E+I+R=1$ holds, that an average duration of the latent state is $1 / \theta$, and that transmission of the infection is 
governed by a incidence rate $f(S, I)$. Then the basic $S E I R$ model is

$$
\begin{aligned}
\dot{S} & =\mu-f(S, I)-\mu S, \\
\dot{E} & =f(S, I)-(\theta+\mu) E, \\
\dot{I} & =\theta E-\delta I .
\end{aligned}
$$

The equation for recovered population, $\dot{R}=\sigma I-\mu R$, is omitted.

The biologically natural condition $f(0, I)=0$ ensures that either of these systems always have an infection-free equilibrium state $Q_{0}$ with the coordinates $S_{0}=1, E_{0}=I_{0}=0$. Apart from this equilibrium, these systems can also have endemic (positive) equilibrium states such that the equalities

$$
\mu-\mu S=f(S, I)=(\theta+\mu) E=B \delta I
$$

where $B=1$ for the $S I R$ model, and $B=(\theta+\mu) / \theta$ for the $S E I R$ model, hold at these states.

It is also useful to note that the octant $(S, E, I) \geq 0$ (quadrant $(S, I) \geq 0)$ is an invariant set of the system (2.2) (system (2.1) respectively).

Now, we proceed to analysis of the global properties of these models.

\section{Global properties of the models}

Global behaviour of these models crucially depends on the basic reproduction number, that is an average number of secondary cases produced by a single infective introduced into an entirely susceptible population. For the $S I R$ and the $S E I R$ models, the basic reproduction 
number is (Driessche and Watmough, 2002)

$$
R_{0}=\frac{1}{B \delta} \frac{\partial f\left(S_{0}, I_{0}\right)}{\partial I}
$$

The following Theorem (Feng and Thieme, 2000, Korobeinikov and Maini, 2005) provides sufficiency conditions for existence of the positive equilibrium states for the systems (2.1) and (2.2).

Theorem 3.1. Let the function $f(S, I)$ be monotonically growing with respect to $S$, and let

$$
\lim _{I \rightarrow 0} \frac{f\left(S_{0}, I\right)}{f(S, I)}>1 \quad \text { for all } S \in\left(0, S_{0}\right)
$$

Then, if $R_{0}>1$, either of these systems, namely (2.1) or (2.2), has positive equilibrium states.

Proof. At a fixed point of the systems (2.1) and (2.2) the equalities

$$
B \delta I+\mu S=\mu \quad \text { and } \quad B \delta I=f(S, I),
$$

where, as above, $B=1$ for the $S I R$ model, or $B=(\theta+\mu) / \theta$ for the SEIR model, hold. These equalities define a negatively sloped straight line $q_{1}$ and a curve $q_{2}$ on the $I S$ plane (Fig. 1). By the implicit function theorem, the monotonicity of the function $f(S, I)$ with respect to $S$ guarantees that the second of these equalities defines a function $S=h(I)$; the curve $q_{2}$ is the graph of this function. This function is positive and either exists and is continuous for $I \in(0, \mu / B \delta)$, or reaches infinity in this interval. In either case, however, it is obvious, that if $S_{*}=h(0) \leq S_{0}=1$, then there is at least one point of intersection of 


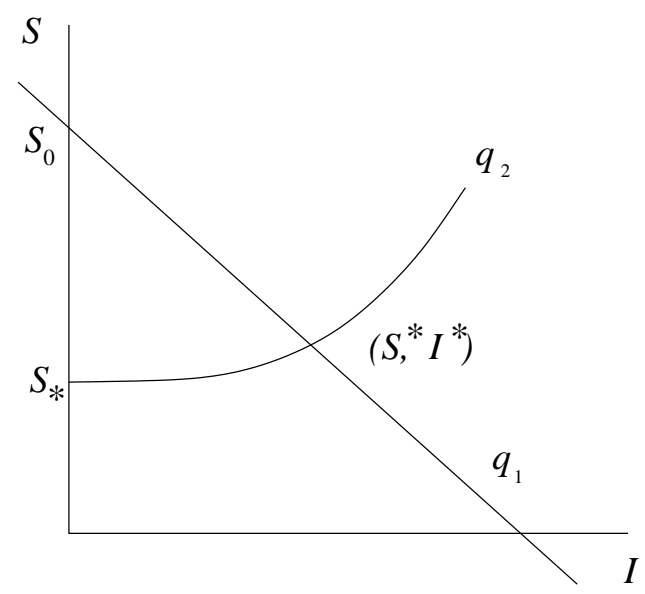

Figure 3.1. The straight line $q_{1}$ and the curve $q_{2}$.

the lines $q_{1}$ and $q_{2}$. By the hypothesis of this theorem, $S_{0} / S_{*}>1$ holds if

$$
\lim _{I \rightarrow 0} \frac{f\left(S_{0}, I\right)}{f\left(S_{*}, I\right)}=\lim _{I \rightarrow 0} \frac{f\left(S_{0}, I\right)}{B \delta I}=\frac{1}{B \delta} \frac{\partial f\left(S_{0}, 0\right)}{\partial I}=R_{0}>1 .
$$

3.1. Global stability of the infection-free equilibrium state for the case $R_{0} \leq 1$. It was mentioned, that the infection-free equilibrium state $Q_{0}$ always exists if $f(S, 0)=0$.

Theorem 3.2. Let

$$
\lim _{I \rightarrow 0} \frac{f\left(S_{0}, I\right)}{f(S, I)}>1 \text { for } S \in\left[0, S_{0}\right), \quad \text { and } \quad \lim _{I \rightarrow 0} \frac{f\left(S_{0}, I\right)}{f(S, I)}<1 \text { for } S>S_{0}
$$

and

$$
f(S, I) \leq I \frac{\partial f(S, 0)}{\partial I_{9}} \text { for all } I>0
$$


Then, if $R_{0} \leq 1$, there is no positive equilibrium state, and the infectionfree equilibrium state $Q_{0}$ is globally asymptotically stable.

Proof. We consider a function

$$
U(S, E, I)=S-\int_{\varepsilon}^{S} \lim _{I \rightarrow 0} \frac{f\left(S_{0}, I\right)}{f(\tau, I)} d \tau+A E+B I
$$

where $\varepsilon$ is a small unspecified parameter, and $A=0, B=1$ for the $S I R$ model, or $A=1, B=(\theta+\mu) / \theta$ for the $S E I R$ model. It is easy to see that $U(S, E, I)$ reaches its global minimum at $Q_{0}$, and, therefore, $U(S, E, I)$ is a Lyapunov function. For the $S I R$ model (2.1) and the $S E I R$ model (2.2), this Lyapunov function satisfies

$$
\begin{aligned}
\frac{d U}{d t} & =\mu-\mu S-\mu \lim _{I \rightarrow 0} \frac{f\left(S_{0}, I\right)}{f(S, I)}+f(S, I) \lim _{I \rightarrow 0} \frac{f\left(S_{0}, I\right)}{f(S, I)}+\mu S \lim _{I \rightarrow 0} \frac{f\left(S_{0}, I\right)}{f(S, I)}-B \delta I \\
& =\mu(1-S)\left(1-\lim _{I \rightarrow 0} \frac{f\left(S_{0}, I\right)}{f(S, I)}\right)+B \delta I\left(\frac{1}{B} \frac{f(S, I)}{\delta I} \lim _{I \rightarrow 0} \frac{f\left(S_{0}, I\right)}{f(S, I)}-1\right) .
\end{aligned}
$$

By the hypothesis of this theorem ,

$$
(1-S)\left(1-\lim _{I \rightarrow 0} \frac{f\left(S_{0}, I\right)}{f(S, I)}\right) \leq 0 \text { for all } S, E, I \geq 0
$$

where strict equality holds only when $S=S_{0}$, and

$$
\frac{1}{B} \frac{f(S, I)}{\delta I} \lim _{I \rightarrow 0} \frac{f\left(S_{0}, I\right)}{f(S, I)}=\frac{1}{B} \frac{f(S, I)}{\delta I} \frac{\frac{\partial f\left(S_{0}, 0\right)}{\partial I}}{\frac{\partial f(S, 0)}{\partial I}} \leq \frac{1}{B \delta} \frac{\partial f\left(S_{0}, I_{0}\right)}{\partial I}=R_{0}
$$

Therefore, $R_{0} \leq 1$ ensures that $\frac{d U}{d t} \leq 0$ for all $S, E, I \geq 0$, where $\frac{d U}{d t}=0$ holds only for $S=S_{0}$. It is easy to verify that the infection-free equilibrium state $Q_{0}$ is the only fixed point of the systems on the plane (line) $S=S_{0}$, and hence, by the Lyapunov-LaSalle asymptotic stability 
theorem (Barbashin, 1970, p. 28, or La Salle and Lefschetz, 1961, p. 58), the equilibrium state $Q_{0}$ is globally asymptotically stable all $S>\varepsilon$ and $E, I \geq 0$. We can extend this statement to the whole non-negative octant (quadrant) observing that the $E I$ plane ( $I$ axis) is transverse to the phase flow, which is directed in the inner of the octant (quadrant), and contains no invariant sets, and then directing $\varepsilon \rightarrow 0$.

The derivative of a Lyapunov function must be equal to zero at an equilibrium state. Since $\frac{d U}{d t}=0$ holds only on the plane $S=S_{0}$, and $Q_{0}$ is the only fixed point on this plane, these systems have no equilibria apart from $Q_{0}$.

\subsection{Stability and uniqueness of the positive endemic equilib-}

rium state. We assume now that the system has a positive equilibrium state $Q^{*}=\left(S^{*}, E^{*}, I^{*}\right)$ such that the equalities

$$
\mu=\mu S^{*}+f\left(S^{*}, I^{*}\right), \quad f\left(S^{*}, I^{*}\right)=(\theta+\mu) E^{*}=B \theta E^{*}=B \delta I^{*}
$$

hold at this steady state.

Theorem 3.3. Let the system (2.1) or (2.2) has a positive equilibrium state $Q^{*}=\left(S^{*}, E^{*}, I^{*}\right)$, such that

$$
\begin{aligned}
& f\left(S, I^{*}\right)<f\left(S^{*}, I^{*}\right) \quad \text { for all } 0<S<S^{*}, \text { and } \\
& f\left(S, I^{*}\right)>f\left(S^{*}, I^{*}\right) \quad \text { for all } S>S^{*},
\end{aligned}
$$


and

$$
\begin{aligned}
& I / I^{*} \leq f(S, I) / f\left(S, I^{*}\right) \leq 1 \quad \text { for } \quad 0<I \leq I^{*}, \text { and } \\
& 1 \leq f(S, I) / f\left(S, I^{*}\right) \leq I / I^{*} \quad \text { for } \quad I \geq I^{*}
\end{aligned}
$$

Then this positive equilibrium state is unique and globally asymptotically stable in $\mathbf{R}_{+}^{n}$ (where $n=2$ or 3 , respectively).

Proof. We consider a function

$V(S, E, I)=S-\int_{\varepsilon}^{S} \frac{f\left(S^{*}, I^{*}\right)}{f\left(\tau, I^{*}\right)} d \tau+C\left(E-E^{*} \ln E\right)+B\left(I-I^{*} \ln I\right)$,

where $B=1, C=0$ for the $S I R$ system $(2.1)$, or $B=(\theta+\mu) / \theta, C=1$ for the $S E I R$ system (2.2). This function is defined and continuous for all $S>\varepsilon$ and $E, I>0$, and satisfies

$\frac{\partial V}{\partial S}=1-\frac{f\left(S^{*}, I^{*}\right)}{f\left(S, I^{*}\right)}, \quad \frac{\partial V}{\partial E}=C\left(1-\frac{E^{*}}{E}\right), \quad \frac{\partial V}{\partial I}=B\left(1-\frac{I^{*}}{I}\right)$.

It is easy to see that the point $Q^{*}=\left(S^{*}, E^{*}, I^{*}\right)$ is a stationary point of the function $V(S, E, I)$ and, by (3.5), it is the only stationary point and the global minimum of this function. Consequently, the function $V(S, E, I)$ is a Lyapunov function. 
For the $S I R$ system (2.1), using (3.4), the Lyapunov function (3.7) satisfies

$$
\begin{aligned}
\frac{d V}{d t}= & \mu-f(S, I)-\mu S \\
& -\mu \frac{f\left(S^{*}, I^{*}\right)}{f\left(S, I^{*}\right)}+\frac{f\left(S^{*}, I^{*}\right)}{f\left(S, I^{*}\right)} f(S, I)+\mu S \frac{f\left(S^{*}, I^{*}\right)}{f\left(S, I^{*}\right)} \\
& +B\left(f(S, I)-\delta I-\frac{I^{*}}{I} f(S, I)+\delta I^{*}\right) \\
= & \mu S^{*}\left(1-\frac{S}{S^{*}}-\frac{f\left(S^{*}, I^{*}\right)}{f\left(S, I^{*}\right)}+\frac{S}{S^{*}} \frac{f\left(S^{*}, I^{*}\right)}{f\left(S, I^{*}\right)}\right) \\
& +f\left(S^{*}, I^{*}\right)\left(1-\frac{f\left(S^{*}, I^{*}\right)}{f\left(S, I^{*}\right)}+\frac{f(S, I)}{f\left(S, I^{*}\right)}\right) \\
& +f\left(S^{*}, I^{*}\right)\left(1-\frac{I}{I^{*}}-\frac{I^{*}}{I} \frac{f(S, I)}{f\left(S^{*}, I^{*}\right)}\right) \\
= & \mu S^{*}\left(1-\frac{S}{S^{*}}\right)\left(1-\frac{f\left(S^{*}, I^{*}\right)}{f\left(S, I^{*}\right)}\right) \\
& f\left(S^{*}, I^{*}\right)\left(3-\frac{f\left(S^{*}, I^{*}\right)}{f\left(S, I^{*}\right)}-\frac{I^{*}}{I} \frac{f(S, I)}{f\left(S^{*}, I^{*}\right)}-\frac{I}{I^{*}} \frac{f\left(S, I^{*}\right)}{f(S, I)}\right) \\
& +f\left(S^{*}, I^{*}\right)\left(\frac{f(S, I)}{f\left(S, I^{*}\right)}-\frac{I}{I^{*}}-1+\frac{I}{I^{*}} \frac{f\left(S, I^{*}\right)}{f(S, I)}\right) .
\end{aligned}
$$

By Theorem hypotheses,

$$
\left(1-\frac{S}{S^{*}}\right)\left(1-\frac{f\left(S^{*}, I^{*}\right)}{f\left(S, I^{*}\right)}\right) \leq 0
$$

(where strict equality holds only when $S=S^{*}$ ), and

$$
\frac{f(S, I)}{f\left(S, I^{*}\right)}-\frac{I}{I^{*}}-1+\frac{I}{I^{*}} \frac{f\left(S, I^{*}\right)}{f(S, I)}=\left(\frac{I}{I^{*}}-\frac{f(S, I)}{f\left(S, I^{*}\right)}\right)\left(\frac{f\left(S, I^{*}\right)}{f(S, I)}-1\right) \leq 0 .
$$

Furthermore,

$$
\frac{f\left(S^{*}, I^{*}\right)}{f\left(S, I^{*}\right)}+\frac{I^{*}}{I} \frac{f(S, I)}{f\left(S^{*}, I^{*}\right)}+\frac{I}{I^{*}} \frac{f\left(S, I^{*}\right)}{f(S, I)} \geq 3
$$


for all $S, I>0$, because the arithmetic mean is greater then or equal to the geometric mean. Therefore, $\frac{d V(S, I)}{d t} \leq 0$ holds for all $S, I \geq 0$, provided that $\mu S^{*}$ and $f\left(S^{*}, I^{*}\right)$ are nonnegative.

In the case of the system (2.2), the Lyapunov function (3.7) satisfies

$$
\begin{aligned}
\frac{d V}{d t}= & \mu-f(S, I)-\mu S \\
& -\mu \frac{f\left(S^{*}, I^{*}\right)}{f\left(S, I^{*}\right)}+\frac{f\left(S^{*}, I^{*}\right)}{f\left(S, I^{*}\right)} f(S, I)+\mu S \frac{f\left(S^{*}, I^{*}\right)}{f\left(S, I^{*}\right)} \\
& +C\left(f(S, I)-(\theta+\mu) E-\frac{E^{*}}{E} f(S, I)+(\theta+\mu) E^{*}\right) \\
& +B\left(\theta E-\delta I-\theta E \frac{I^{*}}{I}+\delta I^{*}\right) .
\end{aligned}
$$

Recalling that $C=1, \mu=\mu S^{*}+f\left(S^{*}, I^{*}\right)$ and $f\left(S^{*}, I^{*}\right)=(\theta+\mu) E^{*}=$ $B \theta E^{*}=B \delta I^{*}$, we obtain

$$
\begin{aligned}
\frac{d V}{d t}= & +\mu S^{*}\left(1-\frac{S}{S^{*}}-\frac{f\left(S^{*}, I^{*}\right)}{f\left(S, I^{*}\right)}+\frac{S}{S^{*}} \frac{f\left(S^{*}, I^{*}\right)}{f\left(S, I^{*}\right)}\right) \\
& +f\left(S^{*}, I^{*}\right)\left(1-\frac{f\left(S^{*}, I^{*}\right)}{f\left(S, I^{*}\right)}+\frac{f(S, I)}{f\left(S, I^{*}\right)}\right) \\
& +f\left(S^{*}, I^{*}\right)\left(1-\frac{E^{*}}{E} \frac{f(S, I)}{f\left(S^{*}, I^{*}\right)}\right) \\
& +f\left(S^{*}, I^{*}\right)\left(1-\frac{I}{I^{*}}-\frac{E}{E^{*}} \frac{I^{*}}{I}\right) \\
= & \mu S^{*}\left(1-\frac{S}{S^{*}}\right)\left(1-\frac{f\left(S^{*}, I^{*}\right)}{f\left(S, I^{*}\right)}\right) \\
& +f\left(S^{*}, I^{*}\right)\left(4-\frac{f\left(S^{*}, I^{*}\right)}{f\left(S, I^{*}\right)}-\frac{E^{*}}{E} \frac{f(S, I)}{f\left(S^{*}, I^{*}\right)}-\frac{E I^{*}}{E^{*} I}-\frac{I}{I^{*}} \frac{f\left(S, I^{*}\right)}{f(S, I)}\right) \\
& +f\left(S^{*}, I^{*}\right)\left(\frac{f(S, I)}{f\left(S, I^{*}\right)}-\frac{I}{I^{*}}-1+\frac{I}{I^{*}} \frac{f\left(S, I^{*}\right)}{f(S, I)}\right) .
\end{aligned}
$$


By Theorem hypotheses,

$$
\left(1-\frac{S}{S^{*}}\right)\left(1-\frac{f\left(S^{*}, I^{*}\right)}{f\left(S, I^{*}\right)}\right) \leq 0
$$

(where strict equality holds only when $S=S^{*}$ ), and

$\frac{f(S, I)}{f\left(S, I^{*}\right)}-\frac{I}{I^{*}}-1+\frac{I}{I^{*}} \frac{f\left(S, I^{*}\right)}{f(S, I)}=\left(\frac{I}{I^{*}}-\frac{f(S, I)}{f\left(S, I^{*}\right)}\right)\left(\frac{f\left(S, I^{*}\right)}{f(S, I)}-1\right) \leq 0$.

Furthermore,

$$
\frac{E^{*}}{E} \frac{f(S, I)}{f\left(S^{*}, I^{*}\right)}+\frac{E}{E^{*}} \frac{I^{*}}{I}+\frac{f\left(S^{*}, I^{*}\right)}{f\left(S, I^{*}\right)}+\frac{I}{I^{*}} \frac{f\left(S, I^{*}\right)}{f(S, I)} \geq 4
$$

for all $S, E, I \geq 0$, because the arithmetic mean is greater then or equal to the geometric mean. Therefore, $\frac{d V}{d t} \leq 0$ holds for all $S, E, I>0$, provided that $\mu S^{*}$ and $f\left(S^{*}, I^{*}\right)$ are nonnegative.

It is easy to see that for both these systems $\frac{d V(S, E, I)}{d t}=0$ holds only when $S=S^{*}$, and that $Q^{*}$ is the only equilibrium state of these systems on this plane (line). Therefore, by Lyapunov-La Salle asymptotic stability theorem (Barbashin, 1970; La Salle and Lefschetz, 1961) the positive equilibrium state $Q^{*}$ is globally asymptotically stable in the positive region $\mathbf{R}_{+}^{n}$ (where $n=2$ or 3 , respectively). The uniqueness of the endemic equilibrium state $Q^{*}$ follows from the fact that the equality $\frac{d V(S, E, I)}{d t}=0$ holds only on the plane (line) $S=S^{*}$, and that the point $Q^{*}$ is the only equilibrium state of the systems on this plane (line). 


\section{SIRS MODEL AND MODELS WITH VERTICAL TRANSMISSION}

For some infections the acquired immunity is not permanent and can disappear after some time. A SIRS model

$$
\begin{aligned}
\dot{S} & =\mu-f(S, I)-\mu S+\alpha R, \\
\dot{I} & =f(S, I)-\delta I, \\
\dot{R} & =\sigma I-(\mu+\alpha) R,
\end{aligned}
$$

where $1 / \alpha$ is an average period of immunity, describes such a case. If $\alpha=0$, then the period of immunity is infinite, and the $S I R S$ model reduces to the $S I R$ model (2.1). Since $S+I+R=1$, the third equation can be omitted.

Moreover, for some infections there is a possibility for a so-called "vertical transmission", that is transplacental transmission from an infective mother to an unborn or newly born child (see Busenberg and Cooke, 1993, for a survey of diseases and models). To incorporate the vertical transmission into the $S I R$ or the $S I R S$ model, we assume that a fraction $p$ of offsprings of the infective hosts is infected at birth, and hence a part of the birth influx, $p \mu I$, enters the infective compartment while the remaining births, $\mu(1-p I)$, come to the susceptibles compartment (Busenberg and Cooke, 1993). Then, using $R=1-S-I$, the $S I R S$ model equations are

$$
\begin{aligned}
\dot{S} & =(\mu+\alpha)-f(S, I)-\alpha I-p \mu I-(\mu+\alpha) S, \\
\dot{I} & =f(S, I)+p \mu I-\delta I .
\end{aligned}
$$


Here the equation for $R$ is omitted. Naturally, $\delta=\sigma+\mu>p \mu$ holds here $(0 \leq p \leq 1)$. If $\alpha=0$, this model reduces to the $S I R$ model

$$
\begin{aligned}
\dot{S} & =\mu-f(S, I)-p \mu I-\mu S, \\
\dot{I} & =f(S, I)+p \mu I-\delta I .
\end{aligned}
$$

The $S I R$ and SIRS models are identical in a certain sense: denoting $\widetilde{\mu}=\mu+\alpha, \widetilde{\delta}=\delta+\alpha=\sigma+\mu+\alpha$ and $\widetilde{f}(S, I)=f(S, I)+(\alpha+p \mu) I$, we obtain the equations

$$
\begin{aligned}
\dot{S} & =\widetilde{\mu}-\widetilde{f}(S, I)-\widetilde{\mu} S \\
\dot{I} & =\widetilde{f}(S, I)-\widetilde{\delta} I .
\end{aligned}
$$

For the $S E I R$ model we also can consider the possibility of the vertical transmission, assuming that fractions of offsprings of the exposed and the infectious hosts, $q$ and $p$ respectively, are infected at birth and, like the hosts infected through the horizontal transmission, enter the exposed compartment (Busenberg and Cooke, 1993). Consequently, there is the influx of the new born into the exposed compartment $\mu(p I+q E)$, and the influx of the new born into the susceptibles compartment is $\mu(1-p I-q E)$ (where, naturally, $0 \leq p, q \leq 1)$. Then the model equations are

$$
\begin{aligned}
\dot{S} & =\widetilde{\mu}-\tilde{f}(S, I)-q \mu E-\widetilde{\mu} S, \\
\dot{E} & =\widetilde{f}(S, I)+q \mu E-(\theta+\mu) E, \\
\dot{I} & =\theta E-\widetilde{\delta} I,
\end{aligned}
$$


where $\tilde{f}(S, I), \widetilde{\delta}$ and $\widetilde{\mu}$ are as defined above with $\alpha=0$ (we use $\widetilde{\delta}$ and $\widetilde{\mu}$ here instead of $\delta$ and $\mu$ for the sake of consistency of notation).

These systems always have an infection-free equilibrium state $Q_{0}=$ $(1,0,0)$ and can have endemic equilibrium states such that the equalities

$$
\widetilde{\mu}-\widetilde{\mu} S=\widetilde{f}(S, I)+q \mu E=(\theta+\mu) E=B \widetilde{\delta} I
$$

hold at these states. For these $S I R, S I R S$ and the $S E I R$ systems with vertical transmission the basic reproduction number is (cf. van den Driessche and Watmough, 2002)

$$
R_{0}=\frac{1}{B \delta}\left(\frac{\partial f\left(S_{0}, I_{0}\right)}{\partial I}+p \mu+q \frac{\delta}{\theta} \mu\right)=\frac{1}{B \delta}\left(\frac{\partial \widetilde{f}\left(S_{0}, I_{0}\right)}{\partial I}+q \frac{\delta}{\theta} \mu-\alpha\right)
$$

where $B=1, q=0$ for the $S I R$ and $S I R S$ models, and $B=(\theta+$ $\mu) / \theta, \alpha=0$ for the $S E I R$ model.

A particular feature of the $S I R S$ model, and the $S I R$ and $S E I R$ models with vertical transmission, which is of importance for further analysis, is that the positive octant (quadrant) of the $S E I$ space (the $S I$ plane) is not an invariant set for these systems. Indeed, it is easy to see that $\dot{S}<0$ at $S=0$ for all $I>1 / p$. Therefore, the problem of proving the global stability for the whole positive domain $\mathbf{R}_{+}^{n}$ (where $n=2$ or 3 respectively) is meaningless for these models. However, the so-called "feasible region"

$$
\Sigma=\left\{(S, E, I) \in \mathbf{R}^{3} \mid S, E, I \geq 0 ; S+E+I \leq 1\right\}
$$


(or $\Sigma=\left\{(S, I) \in \mathbf{R}^{2} \mid S, I \geq 0 ; S+I \leq 1\right\}$ for the $S I R$ and $S I R S$ models) is a positively invariant set, and this is usually considered as the phase space for these systems. Further by the term "global properties" we will imply the properties of these systems in the feasible region $\Sigma$.

Theorems of Section 3 can be extended for these models with the only reservation that by the "global stability" in this case we imply "global stability in $\Sigma "$. It is obvious that if the hypotheses of Theorem 3.1, 3.2 and 3.3 hold for the function $f(S, I)$, then these hold for $\tilde{f}(S, I)$ as well.

Theorem 3.1 can be immediately extended to these models. The only alteration for the proof of Theorem 3.1 is that for the systems (4.1), (4.2) and (4.4) the lines $q_{1}$ and $q_{2}$ are defined by the equalities

$$
B \widetilde{\delta} I+\widetilde{\mu} S=\widetilde{\mu} \quad \text { and } \quad D \widetilde{\delta} I=\widetilde{f}(S, I)
$$

where $B=D=1$ for the $S I R$ and SIRS models, or $B=(\theta+$ $\mu) / \theta, D=(\theta+\mu-q \mu) / \theta$ for the $S E I R$ model. If the hypothesis of Theorem 3.1 holds for the function $f(S, I)$, this holds for $\widetilde{f}(S, I)$ as well, and hence $S_{0} / S_{*}>1$ holds if

$$
\lim _{I \rightarrow 0} \frac{\tilde{f}\left(S_{0}, I\right)}{\widetilde{f}\left(S_{*}, I\right)}=\lim _{I \rightarrow 0} \frac{\tilde{f}\left(S_{0}, I\right)}{D \widetilde{\delta} I}=\frac{1}{D \widetilde{\delta}} \frac{\partial \tilde{f}\left(S_{0}, 0\right)}{\partial I}>1 .
$$

But $\frac{1}{D \widetilde{\delta}} \frac{\partial \widetilde{f}\left(S_{0}, 0\right)}{\partial I}=R_{0}$ for the $S I R$ model, or $\frac{\delta R_{0}+\alpha}{\delta+\alpha}$ for the $S I R S$ model, or $R_{0}+\frac{q \mu}{\theta+\mu-q \mu}\left(R_{0}-1\right)$ for the $S E I R$ model. In either case $R_{0}>1$ suffices to ensure that (4.6) holds. 
Extending of Theorem 3.2 is also fairly straightforward. We consider a Lyapunov function

$$
\widetilde{U}(S, E, I)=S-\int_{\varepsilon}^{S} \lim _{I \rightarrow 0} \frac{\tilde{f}\left(S_{0}, I\right)}{\widetilde{f}(\tau, I)} d \tau+A E+D I,
$$

where $A=0, D=1$ for the $S I R$ and $S I R S$ models, or $A=1, D=$ $(\theta+\mu-q \mu) / \theta$ for the $S E I R$ model. (Note that when $\alpha, p, q=0$, the function $\widetilde{U}$ coincides with the function $U$ used to prove Theorem 3.2). For these models, the Lyapunov function satisfies

$$
\begin{aligned}
\frac{d \widetilde{U}}{d t}= & \tilde{\mu}-q \mu E-\widetilde{\mu} S-\widetilde{\mu} \lim _{I \rightarrow 0} \frac{\widetilde{f}\left(S_{0}, I_{0}\right)}{\widetilde{f}\left(S, I_{0}\right)}+\widetilde{f}(S, I) \lim _{I \rightarrow 0} \frac{\widetilde{f}\left(S_{0}, I_{0}\right)}{\widetilde{f}\left(S, I_{0}\right)} \\
& +q \mu E \lim _{I \rightarrow 0} \frac{\tilde{f}\left(S_{0}, I_{0}\right)}{\widetilde{f}\left(S, I_{0}\right)}+\widetilde{\mu} S \lim _{I \rightarrow 0} \frac{\widetilde{f}\left(S_{0}, I_{0}\right)}{\widetilde{f}\left(S, I_{0}\right)}-D \widetilde{\delta} I \\
= & \widetilde{\mu}\left(1-S-q \frac{\mu}{\widetilde{\mu}} E\right)\left(1-\lim _{I \rightarrow 0} \frac{\widetilde{f}\left(S_{0}, I_{0}\right)}{\widetilde{f}\left(S, I_{0}\right)}\right)+D \widetilde{\delta} I\left(\frac{1}{D} \frac{\widetilde{f}(S, I)}{\widetilde{\delta} I} \lim _{I \rightarrow 0} \frac{\widetilde{f}\left(S_{0}, I_{0}\right)}{\widetilde{f}\left(S, I_{0}\right)}-1\right) .
\end{aligned}
$$

It is obvious that if Theorem hypotheses hold for a function $f(S, I)$, then these hold for $\tilde{f}(S, I)$ as well. Therefore, by the hypothesis of this theorem ,

$$
\left(1-S-q \frac{\mu}{\widetilde{\mu}} E\right)\left(1-\lim _{I \rightarrow 0} \frac{\tilde{f}\left(S_{0}, I_{0}\right)}{\widetilde{f}\left(S, I_{0}\right)}\right)<0 \quad \text { for all } \quad(S, E, I) \in \Sigma
$$

and

$$
\frac{1}{D} \frac{\tilde{f}(S, I)}{\widetilde{\delta} I} \lim _{I \rightarrow 0} \frac{\widetilde{f}\left(S_{0}, I\right)}{\widetilde{f}(S, I)}=\frac{1}{D} \frac{\tilde{f}(S, I)}{\widetilde{\delta} I} \frac{\frac{\partial \widetilde{f}\left(S_{0}, I_{0}\right)}{\partial I}}{\frac{\partial \widetilde{f}\left(S, I_{0}\right)}{\partial I}} \leq \frac{1}{D \widetilde{\delta}} \frac{\partial \widetilde{f}\left(S_{0}, I_{0}\right)}{\partial I} .
$$

But $\frac{1}{D \widetilde{\delta}} \frac{\partial \widetilde{f}\left(S_{0}, 0\right)}{\partial I}=R_{0}$ for the $S I R$ model, or $\frac{\delta R_{0}+\alpha}{\delta+\alpha}$ for the $S I R S$ model, or $R_{0}+\frac{q \mu}{\theta+\mu-q \mu}\left(R_{0}-1\right)$ for the $S E I R$ model. Therefore, $R_{0} \leq 1$ 
ensures that $\frac{d \widetilde{U}(S, E, I)}{d t}<0$ for all $(S, E, I) \in \Sigma$, and hence, by Lyapunov asymptotic stability theorem (Lyapunov, 2002; La Salle and Lefschetz, 1961), the equilibrium state $Q_{0}$ is globally asymptotically stable (in $\Sigma$ ).

To generalise Theorem 3.3 for the models (4.3) and (4.4), we consider a function $\widetilde{V}(S, E, I)=S-\int_{\varepsilon}^{S} \frac{\widetilde{f}\left(S^{*}, I^{*}\right)}{\widetilde{f}\left(\tau, I^{*}\right)} d \tau+C\left(E-E^{*} \ln E\right)+B\left(I-I^{*} \ln I\right)$, where $B=1, C=0$ for the $S I R$ and $S I R S$ systems (4.3), or $B=$ $(\theta+\mu) / \theta, C=(\theta+\mu) /(\theta+\mu-q \mu)$ for the $S E I R$ system (4.4). It is obvious that for $\alpha, p, q=0$ this function coincides with the function (3.7). In the case of the system (4.4), this Lyapunov function satisfies

$$
\begin{aligned}
\frac{d \widetilde{V}}{d t}= & \widetilde{\mu}-\widetilde{f}(S, I)-q \mu E-\widetilde{\mu} S \\
& -\widetilde{\mu} \frac{\widetilde{f}\left(S^{*}, I^{*}\right)}{\widetilde{f}\left(S, I^{*}\right)}+\frac{\widetilde{f}\left(S^{*}, I^{*}\right)}{\widetilde{f}\left(S, I^{*}\right)} \widetilde{f}(S, I)+q \mu E \frac{\widetilde{f}\left(S^{*}, I^{*}\right)}{\widetilde{f}\left(S, I^{*}\right)}+\widetilde{\mu} S \frac{\widetilde{f}\left(S^{*}, I^{*}\right)}{\widetilde{f}\left(S, I^{*}\right)} \\
& +C\left(\widetilde{f}(S, I)-(\theta+\mu-q \mu) E-\frac{E^{*}}{E} \widetilde{f}(S, I)+(\theta+\mu-q \mu) E^{*}\right) \\
& +B\left(\theta E-\widetilde{\delta} I-\theta E \frac{I^{*}}{I}+\widetilde{\delta} I^{*}\right) .
\end{aligned}
$$

Recalling that $\widetilde{\mu}=\widetilde{f}\left(S^{*}, I^{*}\right)+q \mu E^{*}+\widetilde{\mu} S^{*}, \widetilde{f}\left(S^{*}, I^{*}\right)=(\theta+\mu-q \mu) E^{*}$ and $\theta E^{*}=\widetilde{\delta} I^{*}$, and noting that $C(\theta+\mu-q \mu) E^{*}=B \theta E^{*}=(\theta+\mu) E^{*}$, 
we obtain

$$
\begin{aligned}
& \frac{d \tilde{V}}{d t}=\widetilde{\mu} S^{*}\left(1-\frac{S}{S^{*}}-\frac{\tilde{f}\left(S^{*}, I^{*}\right)}{\widetilde{f}\left(S, I^{*}\right)}+\frac{S}{S^{*}} \frac{\tilde{f}\left(S^{*}, I^{*}\right)}{\widetilde{f}\left(S, I^{*}\right)}\right) \\
& +\widetilde{f}\left(S^{*}, I^{*}\right)\left(1-\frac{\tilde{f}(S, I)}{\widetilde{f}\left(S^{*}, I^{*}\right)}-\frac{\tilde{f}\left(S^{*}, I^{*}\right)}{\widetilde{f}\left(S, I^{*}\right)}+\frac{\tilde{f}(S, I)}{\widetilde{f}\left(S, I^{*}\right)}\right) \\
& +q \mu E^{*}\left(1-\frac{E}{E^{*}}-\frac{\tilde{f}\left(S^{*}, I^{*}\right)}{\widetilde{f}\left(S, I^{*}\right)}+\frac{E}{E^{*}} \frac{\tilde{f}\left(S^{*}, I^{*}\right)}{\widetilde{f}\left(S, I^{*}\right)}\right) \\
& +(\theta+\mu) E^{*}\left(\frac{\tilde{f}(S, I)}{\widetilde{f}\left(S^{*}, I^{*}\right)}-\frac{E}{E^{*}}-\frac{E^{*}}{E} \frac{\tilde{f}(S, I)}{\widetilde{f}\left(S^{*}, I^{*}\right)}+1\right) \\
& +(\theta+\mu) E^{*}\left(\frac{E}{E^{*}}-\frac{I}{I^{*}}-\frac{E}{E^{*}} \frac{I^{*}}{I}+1\right) \\
& =\tilde{\mu} S^{*}\left(1-\frac{S}{S^{*}}\right)\left(1-\frac{\tilde{f}\left(S^{*}, I^{*}\right)}{\widetilde{f}\left(S, I^{*}\right)}\right) \\
& +(\theta+\mu) E^{*}\left(1-\frac{\tilde{f}\left(S^{*}, I^{*}\right)}{\widetilde{f}\left(S, I^{*}\right)}+\frac{\tilde{f}(S, I)}{\widetilde{f}\left(S, I^{*}\right)}\right) \\
& +q \mu E\left(-1+\frac{\widetilde{f}\left(S^{*}, I^{*}\right)}{\widetilde{f}\left(S, I^{*}\right)}+\frac{E^{*}}{E} \frac{\tilde{f}(S, I)}{\widetilde{f}\left(S^{*}, I^{*}\right)}+\frac{E^{*}}{E} \frac{\widetilde{f}(S, I)}{\widetilde{f}\left(S, I^{*}\right)}\right) \\
& +(\theta+\mu) E^{*}\left(1-\frac{E^{*}}{E} \frac{\widetilde{f}(S, I)}{\widetilde{f}\left(S^{*}, I^{*}\right)}+1-\frac{I}{I^{*}}-\frac{E}{E^{*}} \frac{I^{*}}{I}\right) \\
& =\tilde{\mu} S^{*}\left(1-\frac{S}{S^{*}}\right)\left(1-\frac{\widetilde{f}\left(S^{*}, I^{*}\right)}{\widetilde{f}\left(S, I^{*}\right)}\right) \\
& +\left((\theta+\mu) E^{*}-q \mu E\right)\left(4-\frac{\tilde{f}\left(S^{*}, I^{*}\right)}{\widetilde{f}\left(S, I^{*}\right)}-\frac{E^{*}}{E} \frac{\tilde{f}(S, I)}{\widetilde{f}\left(S^{*}, I^{*}\right)}-\frac{E}{E^{*}} \frac{I^{*}}{I}-\frac{I}{I^{*}} \frac{\tilde{f}\left(S, I^{*}\right)}{\widetilde{f}(S, I)}\right) \\
& +q \mu E\left(3+\frac{E^{*}}{E} \frac{\widetilde{f}(S, I)}{\widetilde{f}\left(S, I^{*}\right)}-\frac{E}{E^{*}} \frac{I^{*}}{I}-\frac{I}{I^{*}} \frac{\widetilde{f}\left(S, I^{*}\right)}{\widetilde{f}(S, I)}\right) \\
& +(\theta+\mu) E^{*}\left(\frac{\widetilde{f}(S, I)}{\widetilde{f}\left(S, I^{*}\right)}-\frac{I}{I^{*}}-1+\frac{I}{I^{*}} \frac{\widetilde{f}\left(S, I^{*}\right)}{\widetilde{f}(S, I)}\right) .
\end{aligned}
$$


It is obvious that if the hypotheses hold for the function $f(S, I)$, then these hold for the function $\tilde{f}(S, I)$ as well. Hence, by Theorem hypotheses,

$$
\left(1-\frac{S}{S^{*}}\right)\left(1-\frac{\tilde{f}\left(S^{*}, I^{*}\right)}{\tilde{f}\left(S, I^{*}\right)}\right) \leq 0,
$$

where the equality holds only when $S=S^{*}$, and

$$
\frac{\widetilde{f}(S, I)}{\widetilde{f}\left(S, I^{*}\right)}-\frac{I}{I^{*}}-1+\frac{I}{I^{*}} \frac{\widetilde{f}\left(S, I^{*}\right)}{\widetilde{f}(S, I)}=\left(\frac{I}{I^{*}}-\frac{\widetilde{f}(S, I)}{\widetilde{f}\left(S, I^{*}\right)}\right)\left(\frac{\widetilde{f}\left(S, I^{*}\right)}{\widetilde{f}(S, I)}-1\right) \leq 0 .
$$

Furthermore,

$$
\frac{E^{*}}{E} \frac{\tilde{f}(S, I)}{\widetilde{f}\left(S^{*}, I^{*}\right)}+\frac{E}{E^{*}} \frac{I^{*}}{I}+\frac{\widetilde{f}\left(S^{*}, I^{*}\right)}{\widetilde{f}\left(S, I^{*}\right)}+\frac{I}{I^{*}} \frac{\widetilde{f}\left(S, I^{*}\right)}{\widetilde{f}(S, I)} \geq 4
$$

and

$$
\frac{E^{*}}{E} \frac{\widetilde{f}(S, I)}{\widetilde{f}\left(S, I^{*}\right)}+\frac{E I^{*}}{E^{*} I}+\frac{I}{I^{*}} \frac{\tilde{f}\left(S, I^{*}\right)}{\widetilde{f}(S, I)} \geq 3
$$

for all $S, E, I>0$, because the arithmetic mean is greater then or equal to the geometric mean (which is equal to one for both these inequalities). Therefore, $\frac{d V(S, E, I)}{d t} \leq 0$ holds for $S, E, I>0$, provided that $\mu S^{*},(\theta+\mu) E^{*}, q \mu E$ and $(\theta+\mu) E^{*}-q \mu E$ are nonnegative.

However, if $q>0$, then it is possible that $(\theta+\mu) E^{*}<q \mu E$ in $\Sigma$. We assume now that $(\theta+\mu) E^{*}<q \mu E$ holds. Then $\tilde{f}\left(S, I^{*}\right)<\tilde{f}\left(S^{*}, I^{*}\right)$ by $(3.5)$, since $(\theta+\mu) E^{*}=\widetilde{\mu}\left(1-S^{*}\right)$ and

$$
S \leq 1-E-I \leq 1-E \leq 1-q E<1-\frac{\theta+\mu}{\mu} E^{*}=1-\frac{\widetilde{\mu}}{\mu}\left(1-S^{*}\right) \leq S^{*}
$$


for $(S, E, I) \in \Sigma$. Since (3.6) holds for $\widetilde{f}(S, I)$, it follows that

$$
\begin{aligned}
& \frac{d \widetilde{V}}{d t} \leq \widetilde{\mu} S^{*}\left(1-\frac{S}{S^{*}}\right)\left(1-\frac{\tilde{f}\left(S^{*}, I^{*}\right)}{\widetilde{f}\left(S, I^{*}\right)}\right) \\
& +\left((\theta+\mu) E^{*}-q \mu E\right)\left(4-\frac{\widetilde{f}\left(S^{*}, I^{*}\right)}{\widetilde{f}\left(S, I^{*}\right)}-\frac{E^{*}}{E} \frac{\widetilde{f}(S, I)}{\widetilde{f}\left(S^{*}, I^{*}\right)}-\frac{E I^{*}}{E^{*} I}-\frac{I}{I^{*}} \frac{\widetilde{f}\left(S, I^{*}\right)}{\widetilde{f}(S, I)}\right) \\
& +q \mu E\left(3-\frac{E^{*}}{E} \frac{\widetilde{f}(S, I)}{\widetilde{f}\left(S, I^{*}\right)}-\frac{E I^{*}}{E^{*} I}-\frac{I}{I^{*}} \frac{\tilde{f}\left(S, I^{*}\right)}{\widetilde{f}(S, I)}\right) \\
& =\widetilde{\mu} S^{*}\left(1-\frac{\tilde{f}\left(S^{*}, I^{*}\right)}{\widetilde{f}\left(S, I^{*}\right)}\right)-\widetilde{\mu} S\left(1-\frac{\widetilde{f}\left(S^{*}, I^{*}\right)}{\widetilde{f}\left(S, I^{*}\right)}\right) \\
& +(\theta+\mu) E^{*}\left(3-\frac{E^{*}}{E} \frac{\widetilde{f}(S, I)}{\widetilde{f}\left(S, I^{*}\right)}-\frac{E I^{*}}{E^{*} I}-\frac{I}{I^{*}} \frac{\widetilde{f}\left(S, I^{*}\right)}{\widetilde{f}(S, I)}\right) \\
& +\left((\theta+\mu) E^{*}-q \mu E\right)\left(1-\frac{\tilde{f}\left(S^{*}, I^{*}\right)}{\widetilde{f}\left(S, I^{*}\right)}\right) \\
& +\left((\theta+\mu) E^{*}-q \mu E\right)\left(\frac{E^{*}}{E} \frac{\tilde{f}(S, I)}{\widetilde{f}\left(S, I^{*}\right)}-\frac{E^{*}}{E} \frac{\tilde{f}(S, I)}{\widetilde{f}\left(S^{*}, I^{*}\right)}\right) .
\end{aligned}
$$

Recalling that $(\theta+\mu) E^{*}=\widetilde{\mu}\left(1-S^{*}\right)$, we obtain

$$
\begin{aligned}
\frac{d \widetilde{V}}{d t} \leq & (\theta+\mu) E^{*}\left(3-\frac{E^{*}}{E} \frac{\widetilde{f}(S, I)}{\widetilde{f}\left(S, I^{*}\right)}-\frac{E I^{*}}{E^{*} I}-\frac{I}{I^{*}} \frac{\widetilde{f}\left(S, I^{*}\right)}{\widetilde{f}(S, I)}\right) \\
& +\widetilde{\mu}\left(1-S-q \frac{\mu}{\widetilde{\mu}} E\right)\left(1-\frac{\widetilde{f}\left(S^{*}, I^{*}\right)}{\widetilde{f}\left(S, I^{*}\right)}\right) \\
& +\left((\theta+\mu) E^{*}-q \mu E\right) \frac{E^{*}}{E} \frac{\widetilde{f}(S, I)}{\widetilde{f}\left(S, I^{*}\right)}\left(1-\frac{\widetilde{f}\left(S, I^{*}\right)}{\widetilde{f}\left(S^{*}, I^{*}\right)}\right) .
\end{aligned}
$$

Here $S^{*}<1$ for the positive endemic equilibrium state, $\frac{E^{*}}{E} \frac{\widetilde{f}(S, I)}{\widetilde{f}\left(S, I^{*}\right)}+$ $\frac{E I^{*}}{E^{*} I}+\frac{I}{I^{*}} \frac{\tilde{f}\left(S, I^{*}\right)}{\widetilde{f}(S, I)} \geq 3, S+q \frac{\mu}{\tilde{\mu}} E \leq S+E \leq 1$ for all $(S, E, I) \in \Sigma$, and 
$(\theta+\mu) E^{*}<q \mu E$ by the assumption. Therefore, $\frac{d V(S, E, I)}{d t} \leq 0$ for all $(S, E, I) \in \Sigma$.

It is easy to see that for these systems $\frac{d V(S, E, I)}{d t}=0$ holds only when $S=S^{*}$, and that $Q^{*}$ is the only equilibrium state of these systems on this plane (line). Therefore the positive equilibrium state $Q^{*}$ is globally asymptotically stable in the positive region $\mathbf{R}_{+}^{n}$ (where $n=2$ or 3 , respectively). The uniqueness of the endemic equilibrium state $Q^{*}$ follows from the fact that the equality $\frac{d V(S, E, I)}{d t}=0$ holds only on the plane (line) $S=S^{*}$, and that the point $Q^{*}$ is the only equilibrium state of the systems on the plane (line) $S=S^{*}$, where the equality $\frac{d V(S, E, I)}{d t}=0$ holds.

We proved the following Corollary.

Corollary 4.1. Theorems 3.1, 3.2 and 3.3 hold for the systems (4.3) and (4.4) in $\Sigma$.

\section{Discussion AND CONCLUSION}

In this paper, we considered $S I R, S I R S$ and $S E I R$ models of infectious disease where the incidence rate is given by a function $f(S, I)$, which can be nonlinear with respect to both its arguments. We considered global properties of these models and found the sufficient conditions which ensures that these systems are globally asymptotically stable and possess the only globally stable equilibrium state. Depending on the basic reproduction number, this steady state is either the endemic, or the disease-free, so that the infection fades out for $R_{0} \leq 1$. To prove these properties, we used the direct Lyapunov methods: the 
Lyapunov functions which were constructed here made this proof rather simple and elegant.

The sufficient conditions for the global stability of the infection-free equilibrium state $Q_{0}$ for $R_{0} \leq 1$ are (3.2) and (3.3); the sufficient conditions for the global stability of the endemic equilibrium state $Q^{*}$, when it exists, are (3.5) and (3.6) respectively. It is easy to see that the monotonicity of the function $f(S, I)$ with respect to $S$ is sufficient (but not necessary) for existence of the endemic equilibrium state for $R_{0}>$ 1. The conditions (3.2) and (3.5) are for the function $f(\cdot, I)$. These conditions are not very restrictive: monotonic increase of the function $f(S, I)$ with respect to $S$ suffices to ensure that these conditions hold. This implies that the global behaviour of these systems does not depend on the function $f(\cdot, I)$ much. The conditions (3.3) and (3.6) are for the function $f(S, \cdot)$, and these are more restrictive.

It is reasonable to expect that a biologically feasible incidence rate is a function that grows monotonically with respect to both its arguments and is concave, or at least non-convex, with respect to the second argument, $I$; that is $\frac{\partial^{2} f(S, I)}{\partial I^{2}} \leq 0$ for all $S, I \geq 0$. Such concavity may reflect the effects of saturation (possible "self-shading" of the infectives); nonhomogeneity of the environment also generally involved concavity of the incidence rate.

It is readily seen that Theorems hypotheses hold for such a function, and, therefore, we have the following Corollary:

Corollary 5.1. Let the incidence rate $f(S, I)$ be a monotonically increasing with respect to both arguments and non-convex with respect to 
I function Then the systems properties depends on the basic reproduction number $R_{0}$ :

(i) if $R_{0}>1$, then there is an unique and globally asymptotically stable positive equilibrium state $Q^{*}$;

(ii) if $R_{0} \leq 1$, then there is no positive equilibrium state $Q^{*}$, and the infection-free equilibrium state $Q_{0}$ is globally asymptotically stable.

However, we have to stress that this concavity of $f(S, I)$ is not necessary either.

In this paper we postulated that the population size is constant, and that the birth rate exactly equals the death rate for each compartment. However, these constraints can be too restrictive, and they are definitely not necessary for our analysis: these results hold for any model with constant influx rate of new birth and with the transmission rate which does not depend on the recovered population.

These results seem to have important biological implications. It has long been a controversial issue how the system properties depend on a given parametrisation of functional responses. It is indeed an important problem because, in a more applied study, population dynamics models are often used for numerical simulations and that, of course, implies a specific choice of functions. In contrast, we have shown that for a variety of epidemic models, existence and stability of the steady state is robust to the details of the incidence rate dependence on $S$ and $I$, provided biologically feasible properties are held. 


\section{REFERENCES}

Anderson, R.M., May, R.M., 1991. Infectious Diseases in Humans: Dynamics and Control, Oxford University Press, Oxford.

Barbashin, E.A., 1970. Introduction to the theory of stability, WoltersNoordhoff, Groningen.

Briggs, C.J., Godfray, H.C.J., 1995. The dynamics of Insect-pathogen interactions in stage-structured populations. Amer. Naturalist, 145 (6) $855-887$.

Brown, G.C., Hasibuan, R., 1995. Conidial discharge and transmission efficiency of Neozygites floridana, an Entomopathogenic fungus infecting two-spotted spider mites under laboratory conditions. Journal of invertebrate pathology, 65, 10-16.

Busenberg, S., Cooke, K., 1993. Vertically transmitted diseases: Models and Dynamics, Springer, Berlin.

Capasso, V., Serio, G., 1978. A generalisation of the KermackMcKendrick deterministic epidemic model. Math. Biosci. 42, 43-61.

Derrick, W.R., van den Driessche, P., 1993. A disease transmission model in a nonconstant population, J. Math. Biol., 31, 495-512.

Derrick, W.R., van den Driessche, P., 2003. Homoclinic orbits in a disease transmission model with nonlinear incidence and nonconstant population, Discrete and Contin. Dyn. Syst., Ser. B, 3, 299-309.

Feng, Z., Thieme, H.R., 2000. Endemic models with arbitrarily distributed periods of infection I: Fundamental properties of the model, SIAM J. Appl. Math, 61 (3) 803-833. 
Hethcote, H.W., 2000. The mathematics of Infectious diseases, SIAM Rev., 42 (4) 599-653.

Hethcote, H.W., Lewis, M.A., van den Driessche, P., 1989, An epidemiological model with delay and a nonlinear incidence rate, J. Math. Biol., 27, 49-64.

Hethcote, H.W., van den Driessche, P., 1991. Some epidemiological models with nonlinear incidence, J. Math. Biol., 29, 271-287.

Korobeinikov, A., 2006. Lyapunov functions and global stability for SIR and SIRS epidemiological models with non-linear transmission, Bulletin of Mathematical Biology, 68 (3) 615-626.

Korobeinikov, A., Maini, P.K., 2004. A Lyapunov function and global properties for $S I R$ and $S E I R$ epidemiological models with nonlinear incidence, Mathematical Biosciences \& Engineering, 1(1), 57-60.

Korobeinikov, A., Maini, P.K., 2005. Nonlinear incidence and stability of infectious disease models, Mathematical Medicine and Biology: A Journal of the IMA, 22, 113-128.

La Salle, J., Lefschetz, S., 1961. Stability by Liapunov's direct method, Academic Press, New York.

Li, M.Y., Muldowney J.S., van den Driessche, P., 1999. Global stability of SEIRS models in epidemiology, Canadian Appl. Math. Quort. 7.

Liu, W.M., Hethcote, H.W., Levin, and S.A., 1987. Dynamical behaviour of epidemiological models with nonlinear incidence rates, J. Math. Biol., 25, 359-380.

Liu, W.M., Levin, S.A., Iwasa, Y., 1986. Influence of nonlinear incidence rates upon the behaviour of SIRS epidemiological models, J. 
Math. Biol., 23, 187-204.

Lyapunov, A.M., 1992. The General Problem of the Stability of Motion. Taylor \& Francis, London.

van den Driessche, P., Watmough, J., 2002. Reproduction numbers and sub-threshold endemic equilibria for compartmental models of disease transmission, Math. Biosci., 180, 29-48. 